\title{
Therapeutic Bubbles: Basic Principles of Cavitation in Therapeutic Ultrasound
}

\author{
R. Glynn Holt, Ronald A. Roy, Charles R. Thomas, Caleb Farny, \\ Tianming Wu, Xinmai Yang, Patrick Edson \\ Boston University \\ Department of Aerospace and Mechanical Engineering \\ 110 Cummington St., Boston, MA 02215
}

\begin{abstract}
At therapeutic intensities, the application of ultrasound is often accompanied by bubble activity. Bubbles concentrate (by orders of magnitude) acoustic energy and convert that acoustic energy into mechanical, thermal, chemical or frequency-shifted acoustic energy. Depending on the specific clinical context, bubble and cavitation activity may be welcome or unwelcome. Whether desirable or not, certain basic phenomena may be observed, and certain basic principles deduced which can guide researchers in their efforts either to avoid or to exploit cavitation. Towards that end this paper will introduce the fundamentals of bubble dynamics. A bestiary of bubble phenomena and related concepts (such as the Blake threshold, inertial cavitation, shape instabilities, rectified diffusion) will be discussed. The dependence of bubble dynamics on control parameters (acoustic frequency and pressure, pulse parameters) and material properties (shear viscosity, surface tension, temperature, elasticity) will be considered. Experimental techniques and diagnostics for bubble detection and monitoring will be briefly mentioned. Tools for modeling bubble dynamics and their effects will be introduced. Some consequences of bubbles and cavitation activity in vivo (both physical and biological) will be presented, drawing on the example of bubbles in high-intensity focused ultrasound (HIFU) applications. Finally, recommendations of techniques for either avoiding or enhancing (and maybe controlling) cavitation will be proffered.
\end{abstract}

Keywords: HIFU, focused ultrasound surgery, bubble dynamics, cavitation.

PACS: 43.25.Yw; 43.35.Wa; 43.80.Gx; 43.80.Sh

\section{SOME INSTANCES OF BUBBLES IN ULTRASOUND}

The use of high-intensity focused ultrasound (HIFU) to effect focused ultrasound surgery (FUS) is rapidly gaining in popularity. A growing number of pre-clinical studies and clinical trials are under way, and a number of new devices have received FDA approval and/or are on the market. Applications envisioned include transcutaneous and intra-operative hemostasis [1,2], tumor ablation [3], hemolysis [4,5,6,7], drug transport [8], especially in the context of thrombolysis $[9,10,11,12,13,14]$, and the list continues to increase. In all these applications, acoustic cavitation plays a large (sometimes dominant) role in producing the desired effect.

Acoustic cavitation is often the result of the application of high-intensity ultrasound in a biomedical context. There are sound reasons, both experimental and 


theoretical, for defining
therapeutic and diagnostic
operating conditions in order to
minimize bubble activity
$[15,16,17]$. Most of the
arguments boil down to the fact
that bubbles perform a very
efficient and highly nonlinear
conversion of acoustical energy
to mechanical motion. In
therapeutic ultrasound, bubbles
formed in the propagation path
may inhibit deposition of
acoustic energy because they
scatter and absorb much of the
energy before it can reach the
target area, a process often
referred to as 'screening' or
'shielding'.

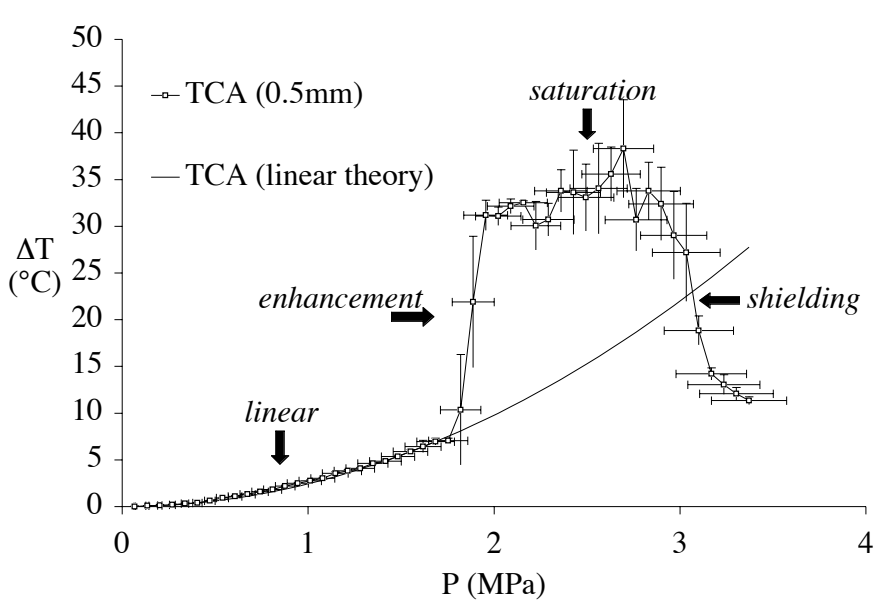

FIGURE 1.1. Peak temperature increase from ambient vs peak-positive acoustic pressure for an agar phantom subjected to $0.7 \mathrm{sec} 1-\mathrm{MHz}$ tonebursts. The thermocouple (TCA) location is measured relative to the acoustic axis. Each data point is the mean of 5 runs at that pressure, with $100 \mathrm{sec}$ cooling between runs.

However, there are therapeutic situations in which it is desirable to excite cavitation. Such is the case in the field of ultrasound surgery using high-intensity focused ultrasound (HIFU), where the goal is to produce irreversible necrosis deep into the tissue with minimal damage in the intervening path (as reviewed in [3], and highlighted in the special issue of IEEE UFFC, November 1996 [18]). If gross mechanical damage and tissue ablation is desired, then excitation of cavitation bubbles is the most efficacious (if also unavoidable) method (for example [19,20,21,22]). Most investigators have argued that cavitation is to be avoided, since it has typically led to unpredictable thermal results [23,24,25]. However, it has been argued [26,27] that with a combination of experiment and numerical modeling of the instability processes, bubble-enhanced heating (observed in [28,29,30,31]) is a controllable and thus desirable modality for HIFU.

Recently several groups [22,32,33) have employed cavitation with therapeutic ultrasound to achieve a variety of clinical ends. At least two groups [34,35] have explicitly initiated cavitation for use in HIFU, noting that cavitation allows them to use lower pressures to achieve the same heating, or that cavitation yields larger lesion sizes for the same pressures.

A key advantage to be gained by actively inducing but controlling cavitation is illustrated in Fig. 1.2. For low pressures ("linear" in the figure), the temperature rise is governed by linear absorption of the primary $1-\mathrm{MHz}$ field, and is linearly proportional to the acoustic intensity, or the square of the acoustic amplitude [36, 37,38]. Above some critical threshold pressure, the heating increases dramatically ("enhancement") coincident with the onset of cavitation. This enhanced heating yields (in the case of Fig. 1.2) approximately a five-fold increase in local temperature rise with respect to the primary heating from the ultrasound alone. As seen in Fig. 1.1, upon increasing 

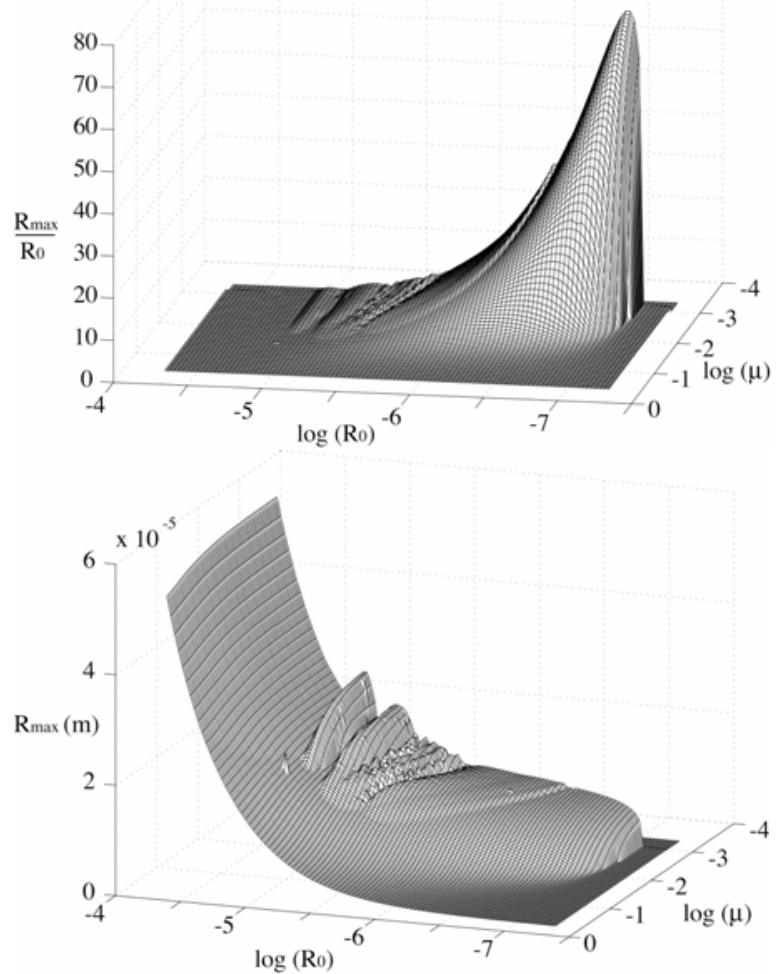

FIGURE 2. Cycle-averaged maximum radius (bottom) and expansion ratio (top) for a single bubble (Eq. 6) driven with $1 \mathrm{MPa}, 1 \mathrm{MHz}$ sinusoidal input. The reference values are 1 micron for $R_{0}$ and 0.001 Pa-s for $\mu$. the pressure at fixed duration of treatment (or equivalently on increasing duration at fixed pressure, not shown) saturation and shielding eventually occur. This shielding regime must be predicted or detected in order to ensure that its disadvantageous effects (unpredictable heating amount and location, and growth of the lesion in the pre-focal region) are minimized or avoided.

\section{A CONSIDERATION OF BUBBLE DYNAMICS}

Space precludes anything like a complete description of bubble dynamics relevant to therapeutic ultrasound. There are several excellent reviews of bubble dynamics per se, many to be found in the text by Leighton [39], itself a wealth of basic bubble dynamics.

Here we have space only to emphasize two points. First, bubbles DO NOT behave like resonant linear oscillators in therapeutic ultrasound. The pressures are too high, and instabilities in shape and size ${ }^{40}$ force bubbles to be much smaller than their resonance size.

Figure 2 gives the reader an overview of the nonlinear radial or breathing mode response as a function of both bubble size and host medium viscosity for a typical pressure of $1 \mathrm{MPa}$ at $1 \mathrm{MHz}$. The expansion ratio plot (top), which also predicts inertial cavitation, has a sharp maximum (referred to [41] as a "giant resonance") on the order of 100 for small $(100 \mathrm{~nm})$ bubbles at low $(0.001-0.01 \mathrm{~Pa}-\mathrm{s})$ viscosities. The maximum radius exhibits several resonance peaks for micron-sized bubbles, and shows a broad and nearly flat plateau for bubbles from 100-1000 nm. In both plots the Blake threshold is clearly seen below $100 \mathrm{~nm}$ for the lowest viscosities. These typical features of radial bubble dynamics will govern their behavior at therapeutic pressures.

Secondly, prediction of bioeffects from therapeutic ultrasound must take into account the nonlinear bubble dynamics. As an example, the lesions formed from HIFU treatments are almost always larger, asymmetric and prefocal compared to the prediction of ultrasound absorption in tissue alone. To predict these asymmetric lesions, a model must incorporate spatially-resolved, time and temperature-dependent bubble dynamics into an effective medium model for the bubbly tissue. This may be 
done by computing effective sound speeds and attenuation coefficients from the numerical solutions to the bubble dynamics equations, and then computing the acoustic propagation using the spatially resolved effective medium model. The results, depicted in Fig. 3, show that only by incorporating the right bubbles can one correctly predict so-called "tadpole-shaped" lesions common in HIFU treatments. There is a striking difference in the lesion shape and position depending on whether there are subresonant bubbles (Type I, left) or superresonant bubbles (Type III, right) in the focal zone. The Type I lesion migrates towards the transducer and appears to be tadpoleshaped. The Type III lesion grows more regularly around the focus, while the Type II lesion is intermediate. The attenuations of sub- and superresonant media are similar but the sound speeds are significantly different, inviting the conclusion that the sound speed change is the dominant mechanism for lesion distortion.

\section{ACKNOWLEDGMENTS}

The authors gratefully acknowledge the financial support of the Department of the Army (award number DAMD17-02-2-0014) and The Center for Subsurface Sensing and Imaging Systems, under the Engineering Research Centers Program of the National Science Foundation (award number EEC9986821).

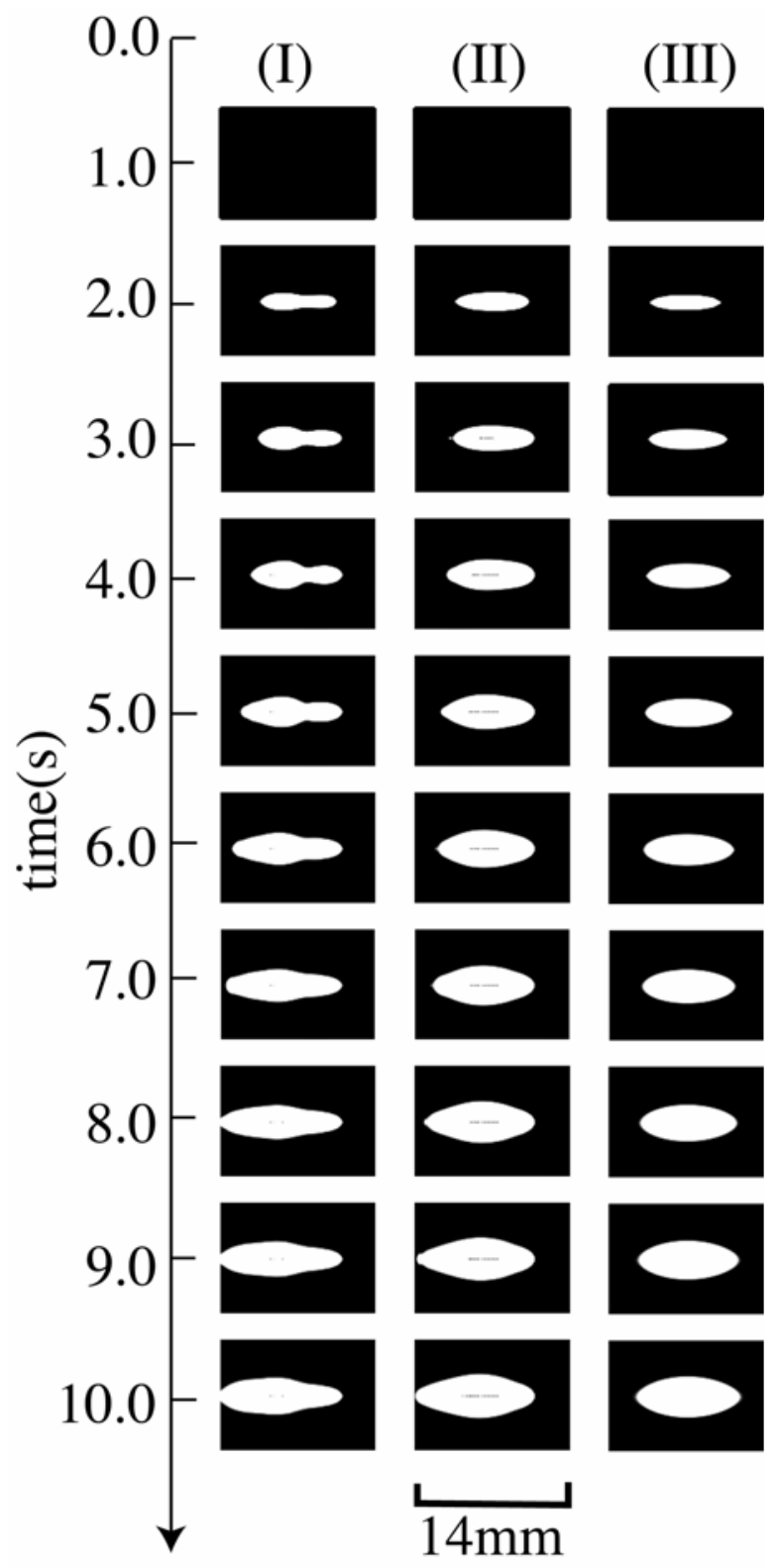

FIGURE 3.15. Thermal lesion development (white values indicate a thermal dose in excess of the threshold defined in the text) plotted at one second intervals from top to bottom. Results are shown for Type I (left), Type II (middle), and Type III (right) bubbles for a 5 second $C W$ HIFU burst propagating from the left at $2 \mathrm{MPa}$ and $1 \mathrm{MHz}$ for a bubble number density of $5 \mathrm{~mm}^{-3}$. Types I, II and III bubble parameters are discussed in the text. 


\section{REFERENCES}

1 Vaezy S, et al. Ultrasound in Medicine and Biology 1997;23:1413-1420.

2 Vaezy S, et al. Echocardiography 2001;18:309-315.

3 ter Haar GR.. Echocardiography 2001;18:317-322.

4 Brayman AA, et al.. Ultrasound in Medicine and Biology 1996; 22: 927-938.

5 Poliachik SL, et al.. Ultrasound in Medicine and Biology 1999; 25: 991-998.

6 Miller DL and Gies RA. Ultrasound in Medicine and Biology 1998; 24: 285-292.

7 Miller MW, , et al. Ultrasound in Medicine and Biology 2001; 27: 709-721.

8 Ng KY, Liu Y. Medicinal Research Reviews 2002;22(2):204-223.

9 Lauer C, , et al. Circulation 1992;86:1257-64.

10 Francis CW, et al . Journal of Clinical Investigations 1992;90:2063-2068.

11 Culp WC, et al. Journal of Vascular and Interventional Radiology 2003;14:343-347.

12 Devcic-Kuhar B, et al . Ultrasound in Medicine and Biology 2002;28:1181-1187.

13 Adler Y, et al . Ultrasound in Medicine and Biology 2003;29:483-486.

14 Alexandrov AV, , et al. Stroke 2002;33:72.

15 Williams A, , et al. Echocardiography 1991;8:423-433.

16 Carstensen EL, et al. Ultrasound in Medicine and Biology 1993;19:147-165.

17 Everbach EC, et al. Ultrasound in Medicine and Biology 1997;23:619-624.

18 IEEE Transactions on Ultrasonics Ferroelectrics and Frequency Control 1996;43: No(6).

19 Fry WJ, et al. Journal of the Acoustical Society of America 1950; 22:867-876.

20 ter Haar GR, et al. British Journal of Cancer 1982; 45 (Suppl. V):151-155

21 Fry FJ, et al. Ultrasound in Medicine and Biology 1996;21:1227-1237

22 Smith NB, et al. Ultrasound in Medicine and Biology 1998;24:1045-1054

23 Chapelon J-Y, et al. Proceedings of the IEEE Ultrasonics Symposium 1991; 1357-1360

24 Chapelon JY, et al. IEEE Transactions on Ultrasonics Ferroelectrics and Frequency Control 1996;43:623-625.

25 Chapelon J-Y, et al. Ultrasound in Medicine and Biology 2000;26:153-159

26 Holt RG, , et al. Ultrasound in Medicine and Biology 2001;27:1399-1412.

27 Holt RG, et al in M.A. Andrews, Crum L, Vaezy S (eds): 2nd International Symposium on Therapeutic Ultrasound. Seattle, WA, American Institute of Physics, 2002, pp 120-131.

28 Lele PP. In Repacholi MH, Grandolfo M, Rindi A, eds. Ultrasound: Medical applications, biological effects and hazard potential. New York: Plenum 1987:275-306

29 Hynynen K. Ultrasound in Medicine and Biology 1991;17:157-169.

30 Sanghvi NT, , et al. IEEE Engineering In Medicine And Biology Magazine 1996;15:83-92.

31 Clarke RL, ter Haar GR. Ultrasound in Medicine \& Biology 1997;23:299.

32 Strickberger SA, , et al. Circulation 1999;100:203-208.

33 Xu Z, , et al. IEEE Transactions of Ultrasonics, Ferroelectrics, and Frequency Control 2004;51:726736.

34 Sokka SD, , et al. Physics in Medicine and Biology 2003;48:223-241.

35 Melodelima D, , et al. Ultrasound in Medicine and Biology 2004;30:103-111.

36 Parker KJ. J. Acoust. Soc. Am. 1983; 74:1356-1361

37 W. L. Nyborg, Phys. Med. Biol. 33 (7), 785-792 (1988).

38 Clarke RL, ter Haar GR. Ultrasound Med. Biol. 1997; 23:299-306

39 Leighton TG. The Acoustic Bubble, Academic Press, 1994.

40 Yang XM, et al. Journal of the Acoustical Society of America 2004;116:3423-3431.

41 I. Akhatov, et al. Phys. Rev. Lett. 78 (2), 227-230 (1997). 